\title{
Flexible Rover Architecture for Science Instrument Integration and Testing
}

\author{
Maria G. Bualat" and Linda Kobayashi ${ }^{\dagger}$ \\ NASA Ames Research Center, Moffett Field, CA 94035-1000, USA \\ and \\ Susan Y. Lee and Eric Park ${ }^{\S}$ \\ Carnegie Mellon University West, Moffett Field, CA 94035, USA
}

\begin{abstract}
At NASA Ames Research Center, the Intelligent Robotics Group (IRG) fields the $\mathrm{K} 9$ and $\mathrm{K10}$ class rovers. Both use a mobile robot hardware architecture designed for extensibility and reconfigurability that allows for rapid changes in instrumentation and provides a high degree of modularity. Over the past several years, we have worked with instrument developers at NASA centers, universities, and national laboratories to integrate or partially integrate their instruments onboard the $\mathrm{K} 9$ and $\mathrm{K} 10$ rovers. Early efforts required considerable interaction to work through integration issues such as power, data protocol and mechanical mounting. These interactions informed the design of our current avionics architecture, and have simplified more recent integration projects. In this paper, we will describe the IRG extensible avionics and software architecture and the effect it has had on our recent instrument integration efforts, including integration of four Mars Instrument Development Program devices.
\end{abstract}

\section{Introduction}

NASA's future robotic missions to Mars and the Moon will attempt to answer questions about their past and present context, the possible existence of life on other planetary bodies, and future habitability of these bodies for humans. The Mars Technology Program, through the Mars Instrument Development Project (MIDP), funds the development and maturation of science instruments for future Mars missions. ${ }^{1}$ The goal of $\mathrm{MIDP}$ is to mature promising instruments from Technology Readiness Level (TRL) 3-analytical and experimental critical function and/or characteristic proof of concept - to TRL 6-system/subsystem model or prototype demonstration in a relevant environment (ground or space). For many of the instruments in MIDP, this involves deployment aboard a rover and testing in a simulated Mars environment.

At NASA Ames Research Center, the Intelligent Robotics Group (IRG) fields the K9 and K10 class rovers. $\mathrm{K} 9$ is a state-of-the-art Mars rover prototype and $\mathrm{K} 10$ is a Lunar rover testbed used primarily for human robot interaction research. Both use a mobile robot hardware architecture designed by the IRG for extensibility and reconfigurability that allows for rapid changes in instrumentation configuration and provides a high degree of modularity.

The extensible hardware architecture is coupled with robust low-level software capable of automatically accommodating changes in hardware configuration, thereby allowing development of instrument control and monitoring code with minimal effort.

The overall architecture addresses the following requirements: a minimum 4 hour run time regardless of payload, fine-grain monitoring and switching of power, the ability to accommodate Mars Exploration Rover (MER) class mobile robots of varying size and speed, and a scalable communications infrastructure.

\footnotetext{
* Deputy Group Lead, Intelligent Robotics Group, MS269-3, Moffett Field, CA 94035

$\dagger$ Electrical Engineer, Intelligent Robotics Group, MS269-3, Moffett Field, CA 94035

₹ Lead Hardware Engineer, Intelligent Robotics Group, MS269-3, Moffett Field, CA 94035

${ }^{\S}$ Computer Engineer, Intelligent Robotics Group, MS269-3, Moffett Field, CA 94035
} 
Over the past several years, we have worked with instrument developers at NASA centers, universities, and national laboratories to integrate or partially integrate their instruments onboard the K9 and K10 rovers. Early efforts required considerable interaction to work through integration issues such as power, data protocol and mechanical mounting. These interactions informed the design of our current avionics architecture, and have made more recent integration efforts much simpler.

Among the instruments we have deployed aboard K9 and/or K10 are:

- CHAMP (Camera HAndlens MicroscoPe) - an arm-mounted focusable microscope capable of color imaging with continuously variable spatial resolution from infinity imaging down to diffraction-limited microscopy.

- A deployable subsurface electromagnetic sounding instrument.

- CMAD (Characterization of Mars Atmospheric Dust) - an atmospheric dust imager and spectrometer.

- MUM (Mars Underground Mole) - a burrowing device that contains a Raman spectrometer.

\section{IRG Rovers}

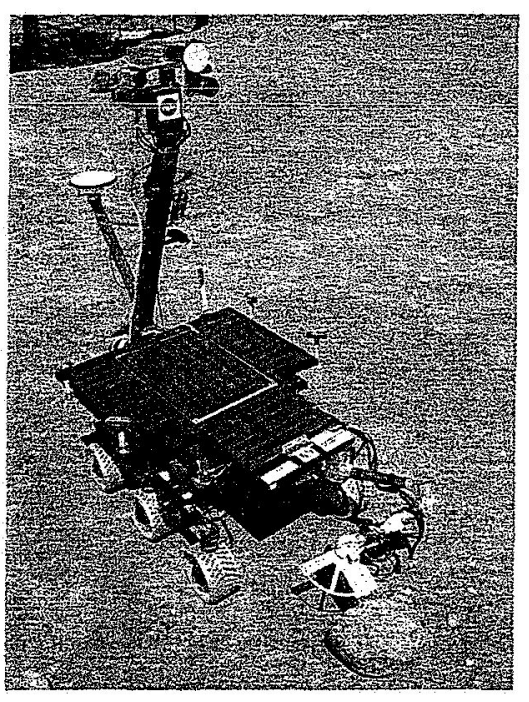

Figure 1. K9 Rover.

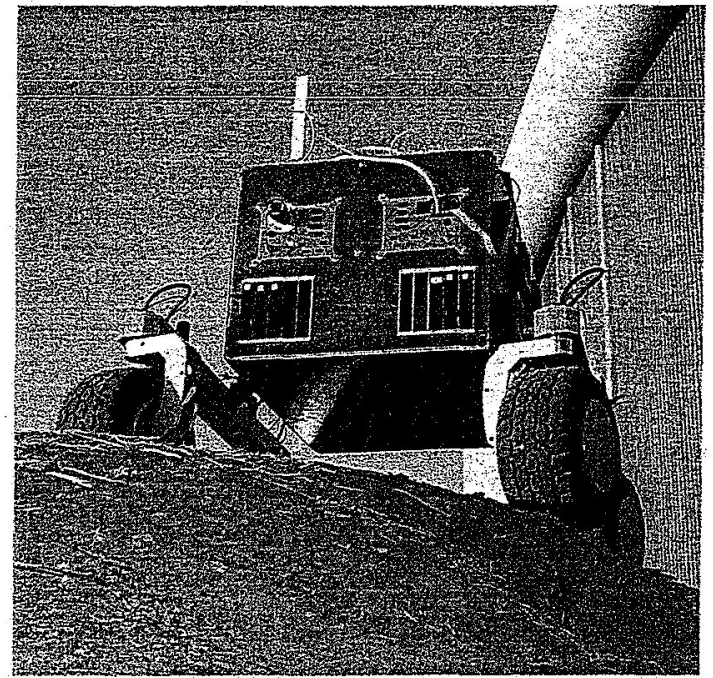

Figure 2. K10 Rover.

\section{A. K9 Overview}

The K9 rover, Fig. 1, is a 6-wheel steer, 6-wheel drive rocker-bogey chassis outfitted with electronics and instruments appropriate for supporting research relevant to remote exploration ${ }^{2-4}$. K9's overall dimensions are $1.05 \mathrm{~m}$ long by $0.85 \mathrm{~m}$ wide by $1.6 \mathrm{~m}$ high, with a mass of approximately $65 \mathrm{~kg}$ and a top speed of approximately $6 \mathrm{~cm} / \mathrm{sec}$. K9's instrumentation suite consists of a Honeywell HMR3000 compass/inclinometer, an Inertial Sciences inertial measurement unit, a Novatel differential global positioning unit, a 5-DOF arm, and four sets of Firewire stereo camera pairs. Two sets of Point Grey Dragonfly cameras mounted under the front solar panel and the rear of the rover provide sensing for hazard avoidance and visualization of the arm workspace. An additional pair of Dragonfly cameras coupled with a pair of Basler A101fc cameras is attached to a pan-tilt unit set atop a rigid mast for navigation and science imaging. Also mounted on the mast is the foreoptic and optical module of a Brimrose acousto-optic tunable filter near-infrared (NIR) spectrometer.

K9's software architecture is based on the Coupled Layered Architecture for Robotic Autonomy (CLARAty) developed at the Jet Propulsion Laboratory in collaboration with NASA Ames Research. Center (ARC) and Carnegie Mellon University. ${ }^{5}$ The use of CLARAty allows us to easily transplant our control software to other CLARAty based robots with no modification to high-level code. 


\section{B. K10 Overview}

K10, Fig. 2, is our next generation mobile robot research platform at ARC. This series of robots is designed to be a cost-efficient, easily maintainable robotic test-bed using as many commercial off-the-shelf components as possible. Each robot features a 4-wheel steer, 4-wheel drive rocker chassis and a top speed of $30 \mathrm{~cm} / \mathrm{sec}$, comparable to human walking speed. Hard points on all sides allow attachment of additional components including antennas, masts, arms, and other equipment.

$\mathrm{K} 10$ 's avionics strives to duplicate those of $\mathrm{K} 9$ wherever possible, including computing infrastructure, power system, and instrumentation. Although the K10 robots draw significantly more power than K9 (approx. 500W versus $100 \mathrm{~W}$ nominal), K9's power architecture is extensible enough to accommodate K10's power requirements with minimal modification, thereby saving engineering time and development costs.

\section{Architectural Overview}

K9's hardware architecture consists of several core avionics elements including a Li-Ion based distributed power system, integrated motion controllers, and a laptop based computing infrastructure, all designed with extensibility and flexibility in mind.

\section{A. Power System}

K9's power system, Fig. 3, has four key elements that allow for easy reconfiguration and expansion: a self-monitoring battery charger board, "smart" Li-Ion battery packs, a power distribution board, and several distributed power switching modules. By creating separate modules within the power system, we developed a modular architecture that can be upgraded or customized incrementally without redesigning the entire system.

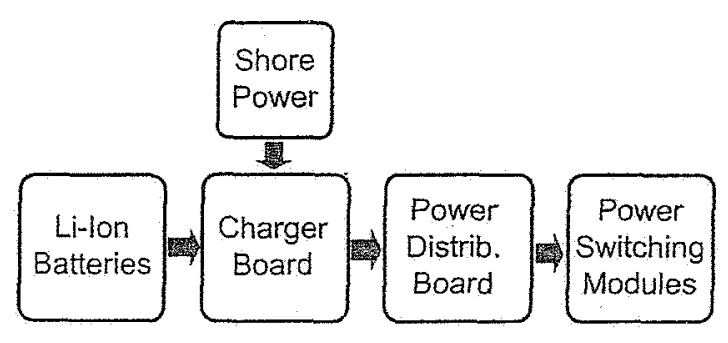

Figure 3. Power System Block Diagram.

\section{Battery Charger Board}

K9's battery charging system consists of a single board capable of supporting the charging and monitoring of up to six hot-swappable battery packs. Several of these in-house designed boards can be stacked in series or parallel to accommodate additional batteries.

The battery charging board uses an Atmel AVR ATMega16L microcontroller and three Linear Technology LTC1960 Li-Ion charging chips. The presence of a microcontroller allows the board to

self-monitor each battery and individually charge them as necessary when external power is applied. While a RS-232 serial interface is provided to allow the host CPU to query battery status, no host intervention is necessary to enable or disable charging. The autonomous nature of the battery charger enables easy expansion by allowing multiple chargers to be present in a robot with no additional supervisory load on the host CPU.

\section{Li-Ion Battery Packs}

K9 carries six Inspired Energy NL2024A22 "smart" battery packs in parallel. Each Li-Ion battery nominally provides $14.4 \mathrm{~V}$ at $6.6 \mathrm{AH}$ and possesses a SMBus interface for easy querying of status and charge capacity. Internal storage of charge state allows the batteries to be hot-swappable, enabling the rover to run continuously without downtime for charging. However, even without hot-swapping, $\mathrm{K} 9 \mathrm{can}$ typically run for a full day on a single charge, far surpassing the 4 hour minimum run time requirement. K10 uses the same battery packs, but three times as many to accommodate the higher current requirements of the drive motors.

\section{Power Distribution Board}

The power distribution board is an in-house designed circuit board used on both $\mathrm{K} 9$ and $\mathrm{K} 10$. Its sole purpose is to take the power provided by the battery charging boards and distribute it to the various onboard instruments. Flexibility is achieved through the use of commercial Vicor DC-DC converters. Power from the battery charging boards is funneled through several Vicor converters which regulate the voltage up or down as necessary before being distributed to instruments. Since these units are available in many different output voltages, it is trivial to mix and match converters as needed. Each power distribution board 
is capable of providing three output voltages but can be extended by stacking boards as necessary to provide additional output voltages or more power per voltage source.

4. Power Switching Modules

Device power switching and fine-grain monitoring requirements are met in a distributed manner using custom USB-enabled power switching modules, Fig. 4. As the number of devices increases, additional power switching modules can be added proportionally. The use of USB as the communications interface allows for a high degree of expandability both in bandwidth and number of supported devices.

On $\mathrm{K} 9$, devices and instruments including the wheels, arm, pan-tilt, compass, and IMU are powered on and off as needed to conserve power when not in use. Since a single USB port can control up to 127 devices and each power switching module can switch power for up to three devices, we can switch up to 381 devices. The addition of a new instrument or change in power requirements merely requires either adding a new power switching module, or changing a few

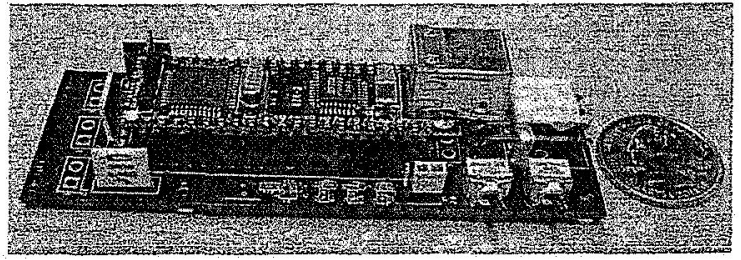

Figure 4. USB-enabled Power Switching Module. passive components on an existing module.

The hot-plug nature of USB interfaces allows for dynamic detection and reconfiguration of power switching modules. Modules placed on the host computer's USB bus are automatically enumerated and made available to $\mathrm{K} 9$. Each module possesses EEPROM memory programmed with the name and position of all devices connected to it. When attached to the computer, an aggregator function presents a list of available devices to the rover control software. If a device of interest appears in the list, a single call to the power switching module class turns on power to the device. Thus, devices can easily be added, removed, and rearranged without requiring any code recompilation or reconfiguration.

5. Power Management

"Smart" batteries, fine-grained control and monitoring of power consumption, and automated charge management allows $\mathrm{K} 9$ to make informed plans given resource constraints ${ }^{6}$. During autonomous operation, given a set of objectives and contingent plans, K9 can choose to perform one set of activities over another set based on its current power consumption, remaining power, and each activity's power requirements. This ability also allows K9 to maximize runtime by turning off unnecessary devices. Use of CLARAty and an extensible power system allowed us to transfer this capability to K10 with no re-engineering of hardware or software.

\section{B. Integrated Motion Controller}

K9 makes use of several six-axis motion controllers, designed at ARC and based on the JR Kerr PICSERVO integrated motor control board design. Each motor control circuit employs a National LMD18200 H-bridge amplifier and the PIC-SERVO/PIC-ENC chipset. Each axis can drive up to 3 amps continuously (6 amps peak at 48VDC max) via pulse width modulation (PWM), accept encoder data, and perform trapezoidal and velocity profiling. Each six-axis board communicates to the host over RS-232 or as part of a RS- 485 bus. By making use of off-the-shelf JR Kerr PIC-SERVO motor control boards, extensibility is restricted only by the number of RS- 485 devices allowed on a single chain. On K9, four six-axis motion controller boards are used to drive six. Maxon drive motors and six Maxon steer motors, a 5-DOF manipulator arm using MicroMo 1319 series motors, and a pan-tilt unit using two Pittman motors. Although initially designed solely to control the drive and steer motors, the board's flexible nature has allowed us to easily expand its use to meet $\mathrm{K} 9$ 's changing motion requirements.

In order to achieve human-walking speeds, K10 uses Pittman 14204S006 Lo-Cog DC Servo Motors. These motors require significantly more power than the 3 amps a single LMD18200 can provide. Instead, $\mathrm{K} 10$ uses readily available Devantech MD03 motor drivers. K10's motion controllers provide an unamplified PWM output which can easily be fed into the PWM input on the Devantech motor driver, allowing us to easily accommodate any size motor with minimal effort.

\section{Computing Infrastructure}

Rapid advances in miniaturization and the development of high-speed serial communications protocols within the consumer computer industry have allowed us to forego PC/104 stacks and CompactPCI cages 
and replace them with powerful subnotebooks and hot-pluggable hubs, both of which allow effortless expansion and reconfiguration.

\section{Host CPU}

The main CPU on our robots consists of an IBM Thinkpad X31 laptop with a $1.4 \mathrm{GHz}$ Pentium M processor. Four cables connect the rover's avionics to its host CPU: one USB cable, one Firewire cable, one Ethernet cable, and an $802.11 \mathrm{~g} \mathrm{RF}$ cable. The USB, Firewire, and Ethernet cables all go into hubs within the electronics enclosure, while the RF cable goes to an $8 \mathrm{~dB} 2.4 \mathrm{GHz}$ antenna. Two Li-Ion laptop batteries provide over six hours of runtime while a PCMCIA $802.11 \mathrm{~g}$ card provides wireless connectivity.

IRG rovers currently run Redhat Linux which was chosen for its large user base and compatibility with other applications. Linux's flexibility and scalability enable us to easily add, remove, and extend devices with minimal difficulty. The robot's controller consists of a single multi-threaded application that runs as a user-space executable. The use of distributed microcontrollers frees us from requiring a real-time operating system, thereby enabling us to dedicate our CPU to navigation, image processing, and other high-level tasks. Fine-grained motor control, power monitoring, and device management are offloaded to the motion control boards, battery charger board, and power switching modules respectively.

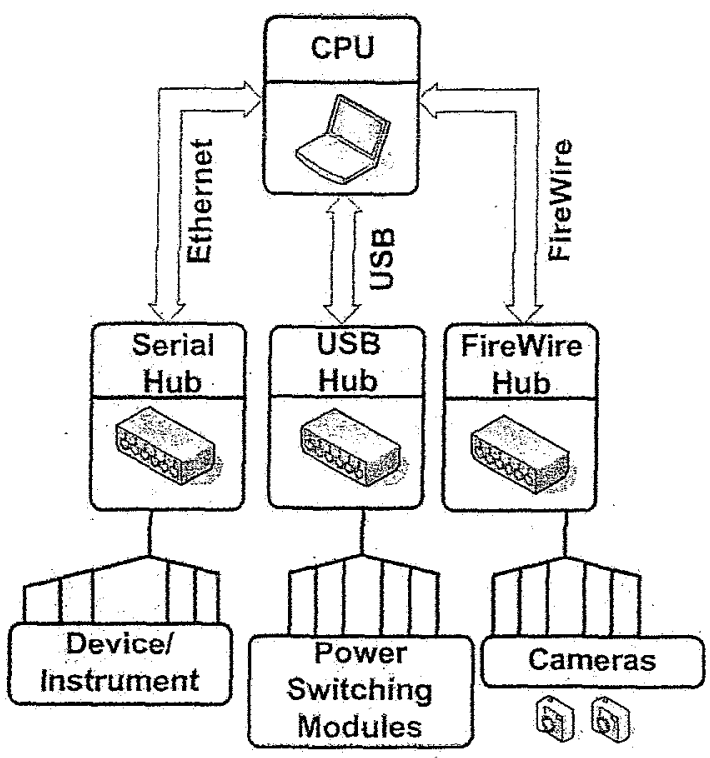

Figure 5. Communications System Block Diagram.

\section{Communications}

Communication across the various devices on $\mathrm{K} 9$ is performed through a myriad of protocols including $\mathrm{I}^{2} \mathrm{C}$, SMBus, RS-232, RS-485, USB, Firewire, and Ethernet. Cables to the host CPU are minimized by employing only high-bandwidth hotpluggable protocols: USB, Firewire, and Ethernet. These high speed interfaces in turn connect to hubs that branch off into multiple low-bandwidth interfaces in a tiered topology as shown in Fig. 5. Firewire is used to interface to the four stereo camera pairs, USB provides connectivity to the power switching boards and other USB based devices, and Ethernet provides communications to serial devices via a Cyclades TS- 800 console server. The TS-800 provides eight serial ports and one Ethernet jack that allows socket, telnet, and ssh connections to the serial ports through the Ethernet interface. Similar to the USB architecture, the use of an Ethernet switch allows us to add an arbitrary number of serial ports as needed. The TS-800 contains a PowerPC processor and also runs Linux, allowing us to easily make modifications and adjust parameters as necessary. The expandable and hot-pluggable nature of Firewire, USB, and Ethernet, along with Linux's support for such protocols satisfies our need for a scalable communications infrastructure that can deal with constantly changing instrumentation payloads.

\section{Integration into K10}

Since publication of Ref. 7, we have built four K10's and have used them in several research projects, ${ }^{8,9}$. The flexible nature of $\mathrm{K} 9$ 's avionics allowed for eased transfer to this new platform. The USB-based power switching modules, Cyclades TS-800 serial-ethernet adapter, and power distribution boards were all integrated into $\mathrm{K} 10$ with no modifications from the system used in $\mathrm{K} 9$,

Differences between $\mathrm{K} 9$ and $\mathrm{K} 10^{\circ}$ s avionics include new commercially available $\mathrm{PC} / 104$ form-factor OceanServer Li-Ion battery charging modules powering 20 Inspired Energy Li-Ion battery packs to replace the custom battery charger board used in K9. Moving to COTS components allows us to reduce development costs while ensuring availability of spares. Additional differences include new $\mathrm{J}$-Kerr Picservo SC motion control boards superseding K9's Picservo v4 boards. The new boards allow on-the-fly changes to position, velocity, and acceleration enabling smooth continuous motion. 


\section{Instrument Integration}

\section{A. CHAMP}

\section{Overview}

CHAMP (Camera HAndlens MicroscoPe) is an arm-mounted focusable microscope capable of color imaging with continuously variable spatial resolution from infinity imaging down to diffraction-limited microscopy ${ }^{10}$. CHAMP, Fig. 6, was developed through the Mars Instrument Development Program (MIDP) by the University of Colorado, Boulder.

Being the first major research instrument to be fully integrated on K9 in March 2002, CHAMP remains a benchmark for mechanical, electrical, and software instrument integration. Prior to CHAMP's integration, a generic palette was designed to hold multiple instruments on the end of K9's arm. The palette consists of a set of eight. evenly spaced holes 90 degrees apart from each other, Fig. 7. K9's 5degree-of-freedom arm allows the instruments on this palette to be pointed at a specific xyz position with defined pitch and yaw angles. Integrating CHAMP mechanically was as simple as informing the University of Boulder team of the spacing requirements (in addition to size and weight limits) and bolting the instrument on the arm.

\section{Integration}

CHAMP is one of several instruments that were integrated prior to K9's distributed electronics upgrade in mid-2004. Because CHAMP did not exist at the time K9's original electronics system was designed, providing CHAMP the power it required was difficult. In order to provide the voltages and power switching capabilities the device required, other instruments had to be disconnected or rerouted so that multiple, unrelated devices were now being switched on and off at the same time. This rewiring of K9's avionics resulted in extremely difficult maintenance and repair of the robot.

It became clear through the integration efforts of CHAMP that we needed to develop a new more distributed electronics architecture in order to keep up with growing instrument integration demands. The redesigned power system architecture consisted of new Li-Ion hot swappable battery packs, a redesigned power distribution system, and distributed power switching modules. With the new power distribution board CHAMP can receive the required $200 \mathrm{~mA}$ at $12 \mathrm{~V}$ and $350 \mathrm{~mA}$ at $5 \mathrm{~V}$ by comnecting to a single connector that is easy to access and clearly labeled, Fig. 8. The old K9 power system had no way to increase the number of power port available; however, with the. new distributed power system more power distribution boards can easily be stacked in the event that more

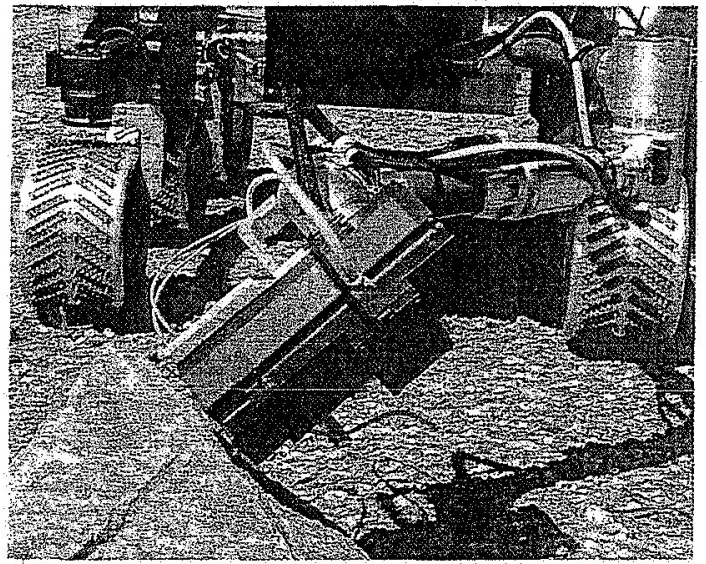

Figure 6. CHAMIP deployed on rock.

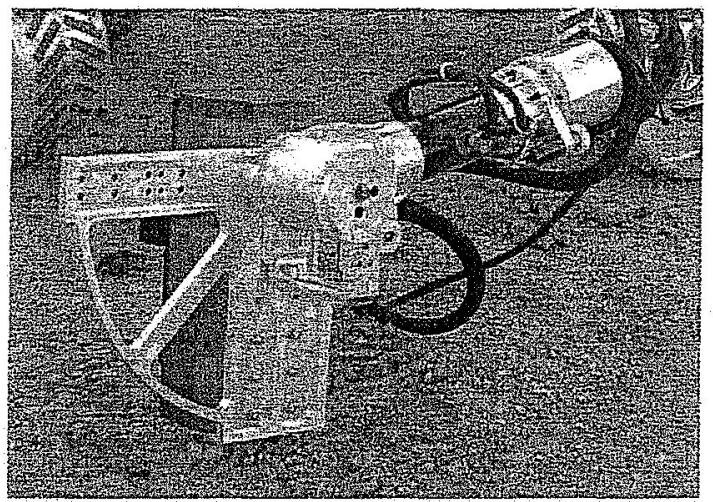

Figure 7. Instrument Palette.

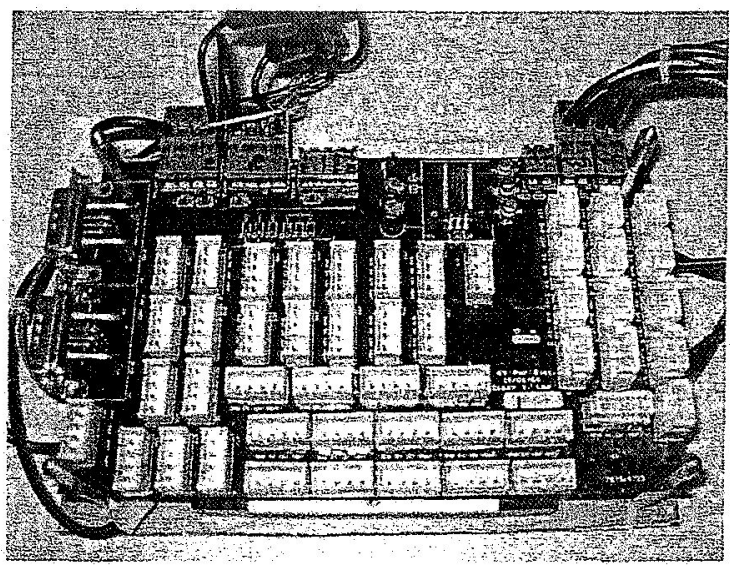

Figure 8. Power Distribution Board. 
than the 49 available ports are needed. In addition, the power switching modules, which allow devices to be turned on and off through a serial interface, can easily-aceommodate-more-devices-by-simply-adding another board or using an available port on an existing board.

CHAMP's need for a reliable RS-232 serial link for communication with the rover also led to an upgrade in K9's communication architecture. Originally, although the CHAMP only required one RS-232 serial link for communication, this link was intermittent due to the inability of most commercial serial hubs to successfully handle tens of devices daisy chained together and then feed into a computer running Linux. Now with our new extensible architecture, $\mathrm{K} 9$ relies on the Cyclades TS-800 to take eight RS-232 ports and turn them into one extremely reliable Ethernet connection to the host computer. When $\mathrm{K} 9$ requires more than eight ports another Cyclades is added and an off-the-shelf Ethernet hub is used to combine the two or more outputs into one.

CHAMP also requires one Firewire interface for its imager. The Firewire ports expand using off-theshelf Firewire hubs.

\section{B. Deployable Subsurface Electromagnetic Sounding Instrument}

1. Overview

Developed by Gregory Delory at the University of California, Berkeley, the deployable subsurface electromagnetic sounding instrument, Fig. 9, is a freestanding instrument package, containing its own power, telemetry, and data processing systems for autonomous operation independent of rover activities. I Its instrument suite consists of subsurface electromagnetic sounders and meteorology sensors. In October 2005, this instrument package was demonstrated on the $\mathrm{K} 9$ rover. While driving, K9 carried the instrument package to a suitable drop location. After deployment, $\mathrm{K} 9$ drove away from the instrument package leaving the electromagnetic sounders to operate free of electrical interference and the meteorology sensors to measure wind, temperature, and properties of the saltation layer without structural or thermal effects by the rover.

\section{Integration}

Two aluminum bars pointing behind K9 were armed with solenoids to hold the instrument payload while $\mathrm{K} 9$ traversed. $\mathrm{K} 9$ deployed the payload by powering the solenoids at $+28 \mathrm{VDC}$ at 4 amps for 1 to 2 seconds.

In the pre-extensible hardware architecture, the single board that handled power switching and power distribution had switches that were assigned fixed input voltages and current ratings. Remote power switching by the rover would have been difficult because the $+28 \mathrm{VDC}$ solenoid voltage requirement was not available on $\mathrm{K} 9$ and therefore no power switches were available with that voltage. In the extensible hardware architecture, power switching modules can be supplied with any input voltage up to 41 volts, the limitation of the Infineon Technologies BTS428L2 MOSFET switches used on the modules. Although $+28 \mathrm{VDC}$ is still not readily available on $\mathrm{K9}$, a power switching module supplied with a $+28 \mathrm{~V}$ battery pack easily allowed remote engagement of the solenoids to release the instrument payload while $\mathrm{K} 9$ drove away.

Thttp://marstech.jpl.nasa.gov/content/detail.cfm?Sect=MTP\&Cat=base\&subCat=MmP\&subSubCat $=$ MIDP H\&Task $\mathrm{ID}=2159$ 


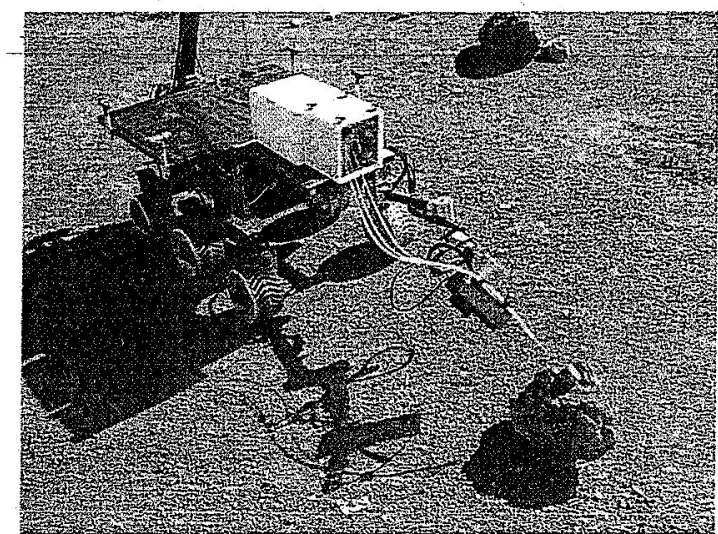

Figure 10. K9 extends the CMAD remote sensor.

\section{CMAD}

I. Overview

CMAD (Characterization of Mars Atmospheric Dust), Fig. 10, is an instrument developed by Philip Jenkins at NASA Glenn Research Center designed to measure the rate of deposition of dust particles and the characteristics of the deposited dust. It performs microscopic imagery of dust and spectroscopy of both atmospheric and settled dust in the visible/near-IR spectrum in an attempt to determine the rate, mechanism, and geographical variation of dust deposition. The instrument itself consists of several miniature spectrometers connected via a fiber bundle deployed on K9's arm and a microscope mounted at the top of the instrument.

\section{Integration}

Communication with the device is made via RS-232 and interfaced to the Cyclades serial-ethernet converter. Power is drawn directly from the $+12 \mathrm{v}$ rail on the power distribution board. As with the other MIDP instruments, electrical integration was easily performed due to readily available power and data connects. At one point, sniffing of the serial traffic between the host computer and the instrument was necessary for interface software development. Rather than requiring a bulky off-board computer to act as an intermediary, we were simply able to utilize an extra Cyclades TS- 800 with the proper in-line cable to read out the bi-directional traffic.

\section{Mars Underground Mole (MUM)}

\section{Overview}

The Mars Underground Mole ${ }^{11}$, Fig. 12, developed by Carol Stoker at NASA Ames Research Center, is a mobile subsurface penetrometer integrated with a Raman spectrometer. Its objective is to deploy and retrieve itself, to burrow to subsurface depths of up to 5 meters, to detect subsurface mineralogy using the Raman spectrometer, and to retrieve a soil sample. As a demonstration of TRL 6, MUM will be fielded on the $\mathrm{K} 10$ rover in September 2006. The mole will be mounted in a stowed position, Fig. 13, while K10 drives to a suitable burrowing site. The deploy and retrieve module (DRM) will unstow the mole's launch tube and payout the tether as the mole hammers itself into the soil. A series of hammering movements within the mole causes forward or reverse momentum, Fig. 11, in soil. As the mole burrows itself, the Raman spectrometer views soil samples through a sapphire window in the hopes of finding mineral species such as phylosillicates,

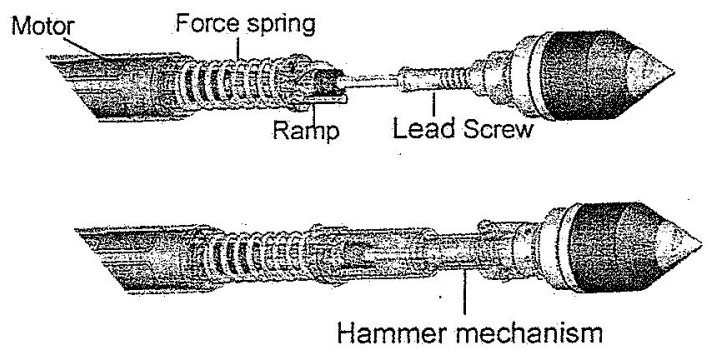

Figure 11. Internal view of MUM showing hammering mechanism. (Image credit: Khoa Nguyen)

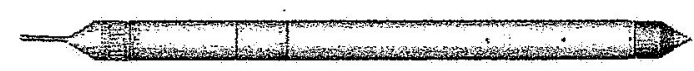

Figure 12. External View of MUM. (Image credit: Hoang $\mathrm{Vu}$ ) sulfates, iron oxides, or carbonates.

\section{Integration}

Using K9's previous avionics architecture, science instrument integration was difficult unless power and computing infrastructure requirements were taken into consideration early on in the instrument design. When instrument integration occurs as an after thought, the powier required by the instrument may not be readily available, power switching capability may be unavailable, and the number of serial communications ports may be limited. Through the use of the extensible hardware architecture, the power distribution board expands or adapts to the needs of the science instrument, power switching and monitoring is provided 
through USB expandable power switching modules, and serial communications expand by adding additional Gyelades serial console servers if one eight port console server is insufficient. MUM takes advantage of these features as follows.

The mole's electronics consists of two Atmel microcontroller based boards that communicate over RS485 serial protocol. One board controls the DRM tether reel motor and the other controls the mole hammering motor. The boards together need $+10-24 \mathrm{VDC}$ at $\max 3 \mathrm{~A}$ for motor power and $+12 \mathrm{VDC}$ at $\max$ $2 \mathrm{~A}$ for electronics power. Since $+12 \mathrm{VDC}$ is a native K10 voltage and all $+12 \mathrm{~V}$ power connections on the power distribution board have not been used, supplying +12 VDC at the maximum MUM current ratings is trivial. If, however, the MUM design team chose to drive the motors at a non-native $\mathrm{K} 10$ voltage, a power distribution board using an appropriately matched Vicor DC-DC converter could be installed into K10.
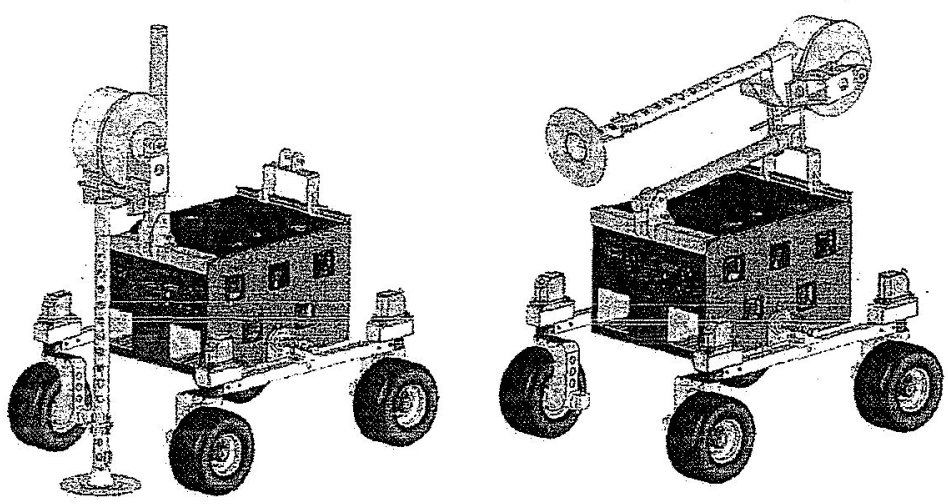

Figure 13. MUM deployed on K10 rover (left), MUM stowed for driving (right). (Image credit: Khoa Nguyen)
A power switching module allows K10 to conserve power while the DRM is in the stowed position. With one output assigned to motor power and another output assigned to electronics power, the power switching module also accommodates current and voltage monitoring while MUM is actively burrowing.

Both microcontroller boards communicate through the rover using a RS-485 to RS-232 converter connected to the Cyclades TS-800 serial console server. Currently, the $\mathrm{K} 10$ rover uses all eight ports on one Cyclades serial console server. Due to the expandable nature of Ethernet through an Ethernet switch, a second Cyclades console server has been added to allow additional serial devices, MUM inclusive, to communicate with the rover.

\section{Conclusion}

NASA's plan to return humans and robots to the Moon, as well as its continuing robotic exploration of the Solar System, requires robots with new autonomy and sensing capabilities ${ }^{12}$. The demand for terrestrial-based analog demonstrations and testbed platforms to prove these technologies is on the rise. With this demand comes the need to produce reliable mobile robots with a proven set of avionics. Drawing upon our experiences integrating commercial instruments, such as navigational sensors and the Brimrose NIR spectrometer, as well as research instruments, such as the MODP instruments described in this paper, we have developed a new hardware architecture that makes optimized use of both off-the-shelf and custom designed hardware in order to achieve flexibility and extensibility. We believe this architecture is capable enough to be used as the basis for a wide variety of current and future mobile robot platforms.

We have created an extensible hardware architecture that can not only be easily adapted and transferred between robot designs, but has also eased instrument integration on our rovers. By taking advantage of the synergistic nature of commercial and custom components, both time and effort have been saved while allowing the architecture to evolve and improve on each implementation.

\section{Acknowledgments}

The authors of this paper would like to acknowledge the support and sponsorship of the Exploration Systems Technology Development, the Intelligent Systems and the Mars Technology programs. We would also like to thank the interns and other members of the Intelligent Robotics Group at NASA Ames Research Center for their continuing support and inspiration. 


\section{References}

${ }^{1}$ Kim, S. S., Hayati, S., Lavery, D., McBride, K. S., "Mars Miniature Science Instruments," IEEE Aerospace Conference, IEEE, New Jersey, 2006, pp. 1 - 11.

${ }^{2}$ Bresina, J., Bualat, M., Edwards, L., Washington, R., Wright, A., "K9 Operations In May '00 Dual-Rover Field Experiment," $6^{\text {th }}$ International Symposium on Artificial Intelligence, Robotics, and Automation in Space, 2001.

${ }^{3}$ Matsuoka, M., Rock, S. M., Bualat, M. G., "Autonomous Deployment of a Self-Calibrating Pseudolite Array for Mars Rover Navigation," IEEE Position Location and Navigation Symposium, IEEE, New Jersey, 2004, pp. 733-739.

${ }^{4}$ Pedersen, L., Sargent, R., Bualat, M., Kunz, C., Lee, S., Wright, A., "Instrument Deployment for Mars Rovers," $7^{\text {th }}$ International Symposium on Artificial Intelligence, Robotics and Automation in Space, 2003.

${ }^{5}$ Volpe, R., Nesnas, I., Estlin, T., Mutz, D., Petras, R., Das, H., "The CLARAty Architecture for Robotic Autonomy," IEEE Aerospace Conference, Vol. 1, IEEE, New Jersey, 2001, pp. 121-132.

${ }^{6}$ Washington, R., Golden, K., Bresina, J., Smith, D. E., Anderson, C., Smith, T., "Autonomous Rovers for Mars Exploration," IEEE Aerospace Conference, Vol. 1, IEEE, New Jersey, 1999, pp. 237-251.

${ }^{7}$ Park, E., Kobayashi, L., Lee, S. Y., "Extensible Hardware Architecture for Mobile Robots," IEEE International Conference on Robotics and Automation, IEEE, New Jersey, 2005, pp. 3084-3089.

${ }^{8}$ Fong, T., et a!, "The Peer-to-Peer Human-Robot Interaction Project," Space 2005, No. AIAA 2005-6750, AIAA, Washington DC, 2005.

${ }^{9}$ Fong, T., et al, "Human-Robot Site Survey and Sampling for Space Exploration," To be published in Space 2006, AlAA, Washington DC, 2006.

${ }^{10}$ Lawrence, G. M., Boynton, J. E., et al, "CHAMP: Camera HAndlens MicroscoPe," $2^{\text {nd }}$ MIDP Conference, Mars Instrument Development Program, TP D-19508, JPL, 2000.

${ }^{11}$ Stoker, C., Lemke, L. G., Gonzales, A. A., "Applications of burrowing moles for planetary and lunar subsurface access," Lunar and Planetary Science 37, Abstract no. 5742, 2006.

${ }^{12}$ NASA, "The vision for space exploration," Tech. Rep. NP-2004-01-334-HQ, NASA, Washington DC, 2004. 